\title{
A Clinical Control Study on the Retroperitoneal Laparoscopy for Unilateral Simple Renal Cyst Decortication in Day Surgery Mode
}

\section{Xiao-Liang Zhu}

WenZhou Medical College Affiliated Taizhou Hospital: Taizhou Hospital of Zhejiang Province

\section{Song-Jiang Wu}

WenZhou Medical College Affiliated Taizhou Hospital: Taizhou Hospital of Zhejiang Province

\section{Yasmeen Bano}

Wenzhou Medical College: Wenzhou Medical University

Wen-Li Liu

Wenzhou Medical College: Wenzhou Medical University

Ming-Hui jiang

Wenzhou Medical College: Wenzhou Medical University

Jia-Hui Liu

Wenzhou Medical College: Wenzhou Medical University

Hai-Hong jiang ( $\nabla$ jianghh.md@foxmail.com )

Wenzhou Medical College First Affiliated Hospital: The First Affiliated Hospital of Wenzhou Medical University

\section{Research article}

Keywords: day surgery, simple renal cyst, retroperitoneal laparoscopy, minimally invasive surgery, medical resource, medical cost

Posted Date: November 24th, 2020

DOl: https://doi.org/10.21203/rs.3.rs-112165/v1

License: (c) (i) This work is licensed under a Creative Commons Attribution 4.0 International License. Read Full License 


\section{Abstract}

Background: Under the constraints of the increasingly tight medical environment and cost, day surgery mode has become a favorable trend and attracts attention from clinicians. Currently, there are no standards or procedures on the retroperitoneal laparoscopy for unilateral simple renal cyst decortication in day surgery mode. We attempted to investigate the feasibility and safety criteria of unilateral simple renal cyst decortication by retroperitoneal laparoscopy in the day surgery ward and to compare the advantages and the disadvantages with conventional inpatient ward mode.

Methods: A total of 41 patients with unilateral simple renal cysts meeting surgical indications were enrolled in this study. To see whether patients in the day group could successfully complete the procedure and be admitted and discharged within 24 hours and to summarize the advantages and the advantages. The indexes of the operation time, time of hospitalization, postoperative complication rate, total costs of hospitalization and other indicators were compared between the two groups.

Results: 41 cases of unilateral simple renal cyst decortication were completed successfully. Patients were followed up 10-12 months after surgery. The results of time of hospitalization, total costs of hospitalization and time of postoperative removal of drainage tube were statistically significant between the two groups of the day ward group and the inpatient ward group $(P<0.05)$.

Conclusions: We concluded the retroperitoneal laparoscopy for unilateral simple renal cyst decortication in patients with unilateral renal cysts was safe and feasible in day surgery mode under certain indicative criteria. The criteria included patients' age between 40 and 80 years, no previous history of abdominal surgery, CT showing a unilateral renal cyst (Bosniak I or II), preoperative ASA assessment was I or II, no contraindications to surgery or anesthesia, postoperative team management and discharge assessment. Advantage of reducing time of hospitalization and total medical cost may relieve the situation of the shortage of medical resources.

\section{Background}

The concept of day surgery ward was first proposed by James Nicoll, a famous British pediatric surgeon in 1909. It was defined as selecting the appropriate patients to complete hospitalization, surgery, shortterm observation, recovery and discharge within 1 working day [1]. The United States established its first independent ambulatory surgery center as early as in 1970, and many other countries began to accept and promote ambulatory surgery. The International Association for Ambulatory Surgery (IAAS) defined day surgery in 2003 as a procedure performed by a patient on admission, surgery, and discharge in one working day, excluding doctor-type clinics or hospital outpatient-type surgeries [2]. About surgical treatment of renal cysts in practice, most domestic hospitals in China applied laparoscopic renal cyst decortication surgery under inpatient ward mode, which had the disadvantages of long hospital stay and high hospital-related costs. Currently, there is no clinical control study on the operation of renal cyst in the 
day ward model. To explore its feasibility, safety and comparison with the traditional model, we conducted a single-center case-control study for comparisons.

\section{Methods}

\subsection{Study population}

During the period from 15 January 2018 to 15 March 2019, 49 patients with renal cyst with surgical indications were enrolled in the urology outpatient clinic. A total of 41 patients were included in this clinical control study and 8 patients were excluded based on exclusion criteria. The entire process was shown in the flow diagram of study design and participants (Fig. 1). The included patients were randomized to the day ward group (19 patients) and the inpatient ward group (22 patients).The patients of day ward group completed all preoperative examinations in the outpatient department and completed admission, operation and discharge within 24 hours. The surgical indication was the maximum diameter of simple renal cyst more than 4 centimeter and presence of obvious renal parenchymal compression and damage on imaging studies, or there were clinical symptoms such as waist swelling, hematuria and

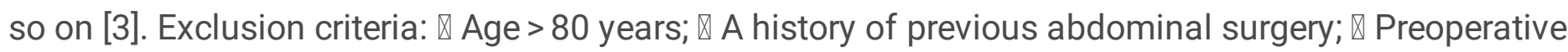
American Society of Anesthesiologists(ASA) grade was III or IV, or contraindications to surgery or

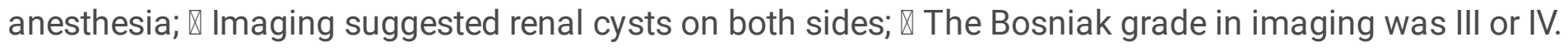
Inclusion criteria were summarized as follow: age between 40 to 80 years old, no previous history of abdominal surgery, unilateral renal cysts (Bosniak I or II) on Computed Tomography (CT), preoperative ASA assessment was I or II, no surgical or anesthetic contraindications.

\subsection{Preoperative examination}

All patients completed the following preoperative examinations: $₫$ laboratory tests: blood routine, blood type, clotting function, complete biochemistry, tumor series, Human Immunodeficiency Virus (HIV), syphilis, hepatitis $B$, hepatitis $C$, urinary routine, fecal routine; $₫$ imaging tests: urological ultrasound, CT enhancement and chest X-ray; $\otimes$ electrocardiogram; $\otimes$ special tests: cardiopulmonary function and lower limb vascular ultrasound if the patient was older than 60 years. Patients in the day ward group completed the above examinations in the outpatient department, and the outpatient anesthesiologist assessed the ASA grade of the patients.

\subsection{Surgical procedures}

All operations were performed by a surgeon who had experience of 15 years and above in urology. General anesthesia was performed under the condition of endotracheal intubation. In brief, the 6 steps of surgical procedure were described as follows. Step 1: the patient was placed in the lateral position, followed by the establishment of three trocar tunnels. Step 2: The first Trocar of the puncture point was located at a transverse incision of $1.0 \sim 1.5 \mathrm{~cm}$ under the twelve costal margin of the posterior axillary line. The second puncture point was located at a transverse incision of $1.0 \sim 1.5 \mathrm{~cm}$ above the iliac crest in the middle axillary line, and then a $10 \mathrm{~mm}$ trocar was placed for the placement of the observing lens. 
The third puncture point was located under the costal margin of the axillary front line. A transverse incision of about $0.5 \mathrm{~cm}$ was made with the placement of a $5 \mathrm{~mm}$ trocar. Step 3: $\mathrm{CO}_{2}$ was injected into the retroperitoneal cavity to establish artificial pneumoperitoneum after above placement. Step 4: The psoas major, perirenal fat sac and other anatomical markers were carefully identified through the trocar tunnels. The location of the cyst was determined combining with preoperative imaging. The cyst was cut open and cyst fluid was sucked out clean. The cyst wall was lifted and removed approximately $5 \mathrm{~mm}$ from the edge of renal parenchyma. Step 5: A drainage tube with negative pressure was placed after removing the cyst with no bleeding. The puncture hole was then sutured after removing the gas from retroperitoneal cavity. Step 6: The removed renal cyst was sent for pathological examination.

\subsection{Discharge assessment and criteria}

Patients were evaluated by the surgeon, anesthesiologist, and nurse for postoperative pain, drainage of the tube, and recovery of the bowel function. All patients were discharged from the hospital with meeting of following criteria: $\otimes$ The patient's gastrointestinal function was restored, the patient's anus was ventilated, there was no discomfort after eating semi-fluid food, and patients could get out of bed by themselves. $\otimes$ The patients' vital signs were stable with soft abdomen. There was no obvious abdominal pressure pain or muscle tension. $\square$ No obvious decreased hematocrit on blood tests and no obvious

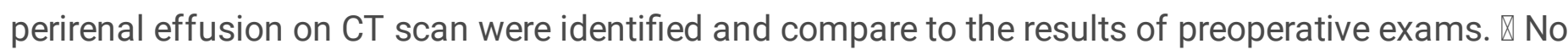
related significantly postoperative complications were found after removing drainage tube before patient discharge. $\otimes$ The patient was accompanied by an adult dependent before discharge.

\subsection{Clinical related data Collections}

Related clinic data of all 41 patients were collected, including their basic characteristics, imaging results, surgical procedure-related data, and follow-up data. The main comparisons between two groups were conducted including operation time (time in minutes, from beginning surgical incision to its closure), duration of hospitalization (time in hours), intra-operative blood loss which was evaluated by both surgeon and anesthesiologist based on the volume of suction collection, in milliliter), time of postoperative drainage tube removal (time in hours, from insertion of drainage tube to removal of drainage tube), the incidence of postoperative complications (incision exudation, incision infection and urinary tract infection), the total costs of hospitalization (RMB), the postoperative patients' satisfaction rate (\%), preoperative and postoperative patients' quality of life scores, renal cyst recurrence after surgery, etc.

All patients were followed up 10-12 months after procedure. The follow-up data included the images of renal cyst, recurrence of cysts or remission of hydrocele, and the questionnaires on quality of life, satisfaction rate and etc.

\subsection{Statistical analysis}

Statistical software spss 19.0 was used for statistical analysis. Mean \pm standard deviation $(x \pm s), n(\%)$, $\mathrm{n} / \mathrm{N}(\%)$ were applied to represent the results of the data. The t-test was used for data of measurement, 
and the $\chi 2$ test was used for data of count. When $p<0.05$, the difference was considered to be statistically significant.

\section{Results}

\subsection{Results of patients' clinical data.}

The data collected from 41 patients includes: clinical characteristics (table 1), imaging characteristics (table 2), surgery-related data (table 3), and the follow-up data (table 4). The preoperative imaging findings of 41 patients indicated the maximum diameter of all renal cysts were over $4 \mathrm{~cm}$ accompanying varying degrees of renal parenchyma compression and structure damage. All patients' unilateral simple renal cysts were decorticated successfully under retroperitoneal laparoscopy with no opening surgical procedures or major bleeding during the operation. All patients' cystic fluid was clear except for one patient with cloudy cystic fluid. The drainage tube was removed before patient discharge without substantial complications, such as urine extravasation or massive bleeding required blood transfusion.

There were significant differences between the day ward group and the inpatient ward group $(P<0.05)$; including the mean time of postoperative removing the tube $(9.7 \pm 1.8$ vs $20.8 \pm 5.5)$ hours, the mean total costs of hospitalization $(5790 \pm 99.8$ vs $7496 \pm 120.6) \mathrm{RMB}$, the mean time of hospitalization $(22.7 \pm 1.4$ vs $46.4 \pm 11.3$ )hours.

\subsection{Results of surgical complications}

Complications occurred in a total of $4(9 \%)$ patients; including obvious postoperative incision exudation in 2 patients (5\%), postoperative incision infection in 1 patient $(2 \%)$, and postoperative urinary tract infection in 1 patient (2\%). For obvious incision exudation standard, we included the patients with gauze use for exudation over 5 pieces and over 3 times incision dressing change after the procedure. The postoperative urinary tract infection was identified to be staphylococcus aureus in cultures with recovery by oral antibiotic treatment. For postoperative urinary tract infection patient with urine frequency and urine pain, the routine urinalysis indicated white blood cell (++) but no fever and no positive urine culture. The patient was relieved of the symptoms with negative result of urinalysis 1 week after discharge.

\subsection{Results cyst of pathology}

All removed cyst tissue specimens were sent to pathological department for histopathological examination, which all confirmed benign renal cyst wall.

\subsection{Results of postoperative follow-up}

CT scan or ultrasound examination was used to observe the change of kidney to exclude hydronephrosis or recurrence of renal cyst after renal cyst decortication procedure. All the patients underwent CT or ultrasound examination, indicating the original renal cyst had been surgically decorticated with no recurrence of the cyst at the time of 10-12 months follow-up. Regarding satisfaction rate and quality of life (QOL) scores at 2 weeks after procedure, the inpatient ward group was slight higher than the day ward 
group; however there was no significant difference. Ultrasound examination 2 weeks after procedure indicated that all 3 patients with mild hydronephrosis before procedure were relieved. All 10 patients with preoperative bulging pain around the waist were relieved after 3 months of postoperative follow-up. Hematuria disappeared in 3 out of 4 patients with preoperative hematuria, who received exfoliative cytology but no suspicious malignant cells, remaining 1 patient with positive continuous hematuria refused further invasive examinations suggestion, such as cystoscopy, but still in long-term follow-up at our urology outpatient clinic.

\section{Discussion}

Renal cyst has become popular in the population due to the development of imaging technology and health insurance on screening $[4,5]$. Renal cyst is usually one of most common medical conditions with no symptoms. Only a few patients have symptoms such as pain, hypertension, hematuria, recurrent urinary tract infection and even cyst rupture [6, 7]. According to Bosniak classification, the renal cysts can be classified as simple cysts (Bosniak grade I and II) or complex cysts (Bosniak grade III and grade IV). In practice for patients with simple renal cyst (Bosniak grade I and grade II), if the maximum diameter of cyst is less than $4 \mathrm{~cm}$ with no obvious clinical symptoms, the patient is usually advised regular follow-up. If the maximum diameter of the renal cyst is over $4 \mathrm{~cm}$ with obvious renal parenchymal damage on imaging, and with clinical symptoms and hematuria, the patient can be offered to undergo surgical removal. It aims to prevent further progression of the disease or further potential damage to the kidney function. There are many treatment options, such as ultrasound-guided puncture drainage plus hardener agent injection [8], traditional open surgery for decortication of renal cyst, laparoscopic decortication renal cyst surgery, flexible ureteroscopic management of renal cyst $[9,10]$ and percutaneous decortication renal cyst surgery [11].

Laparoscopic renal cyst decortication has the characteristics of less trauma, faster recovery, and better efficacy. It has become a preferred treatment option, especially for young patients with large cyst [12]. A comparative study between retroperitoneal approach and peritoneal approach demonstrated the retroperitoneal approach has significantly shorter operative time than the peritoneal approach. Moreover, the retroperitoneal approach may reduce the interference to the intestinal tract with significantly shorten the recovery time of intestinal function [13]. With the concepts of accelerated rehabilitation surgery and postoperative pain-free surgery developing in practice, the ambulatory surgery has become popular in hospital because of its simplified and standardized process of admission, diagnosis, treatment, and discharge. It seems can also relief the shortage of medical resource effectively [14, 15]. In the study, postoperative bowel function was restored before discharge in the day ward group. We attributed to the retroperitoneal approach protecting the peritoneum and the standardized cooperation process from the postoperative management team. In the case of infected cysts, the retroperitoneal approach may also reduce the incidence of peritoneal spread $[16,17]$.

The infections of catheter-related urinary tract had been reported increasing with each additional day of catheter retention [18]. Since the hospital stay in day surgery would not exceed 24 hours with the increase 
of the turnover rate; it may reduce the indwelling time of urinary catheters, and may also reduce the incidence of nosocomial infections. If postoperative infection occurs, an appropriate antibiotic can be prescribed in outpatient clinic according to the results of bacterial culture from samples. Including the postoperative complication rate from our study, there is no significant difference between two groups in comparison of the surgery related outcomes (operation time, intraoperative blood loss, 10-12 months of postoperative follow-up for renal cyst recurrence, postoperative quality of life score, and postoperative satisfaction rate). However, both the total hospitalization time and the time of postoperative drainage tube indwelling are significantly shortened, which reduces the medical expenses and health resources need.

Appropriate patient candidate selection and adequate preoperative assessment and preparation can be critical for prerequisites for the success of the day surgery $[19,20]$. Therefore, for the patient safety, our procedures of laparoscopic decortication were tested in unilateral simple renal cyst under following requirements: $₫$ The operator has skilled to the procedure with over 15-year experiences. $\square$ All patients'

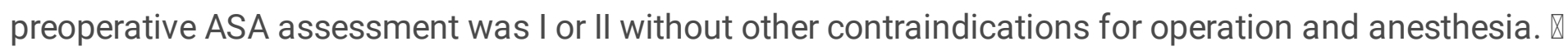
Their Bosniak grades was restricted in the categories of I or II. Furthermore, the location, size and number of renal cysts as well as the relationship between renal cysts and peripheral vessels and renal collecting system were carefully analyzed by the operator according to preoperative ultrasound and CT examination. $\otimes$ The standardized cooperation process protocols from the postoperative management team can timely assess postoperative pain, intestinal function, and drainage. $\otimes$ The postoperative management team scheduled postoperative exam process timely, like CT scan and blood test. $\nabla$ Every postoperative patient was evaluated by the team at the time of discharge according to enhanced recovery after surgery criteria. Antecedents to recovery, the time required to recover, support and encouragement, regular appropriate information, and setbacks during recovery were all thought to affect the recovery process in positive or negative directions.

This study has several limitations. It was a prospective investigation, a single-center and case-controlled study. All cases were limited to simple and unilateral renal cyst cases with several other limitations, we cannot conclude if it is still safe and effective in other situations, like bilateral renal cyst, other Bosniak grades, or even complicated renal cyst under day surgery mode. Therefore, further investigations and large-size study may be required to confirm if patients with surgically-indicated renal cysts have benefit from the day ward mode.

Therefore, we suggested retroperitoneal laparoscopic unilateral simple renal cyst decortication is safe and feasible in the day ward model for patients who have appropriate conditions according to the inclusion standards in our study. It may reduce the need of our limited medical resources, reduces the possibility of urinary tract infections, and shortens patient's hospital stay. Day surgery is considered to be a high-quality, safe, and cost-effective surgical mode, which requires clinicians to continuously explore and accumulate evidences in practice. During the implementation of day surgery mode, we believe it can improve medical quality with reducing medical costs in renal cysts in ambulatory surgery, which eventually benefits both patients and clinicians. In addition, for the current prevention and control of the 
COVID-19 pandemic, day surgery mode could be one of the best options suitable for patients with reduced hospital-stay time and protective supplies cost.

\section{Conclusions}

From the study we demonstrated retroperitoneal laparoscopic renal cyst decortications ambulatory surgery was safe and feasible in patients with unilateral cyst under certain indicative criteria. The criteria included patients' age between 40 and 80 years, no previous history of abdominal surgery, CT showing a unilateral renal cyst (Bosniak I or II), preoperative ASA assessment was I or II, no contraindications to surgery or anesthesia, postoperative team management and discharge assessment. With the development of minimally invasive techniques, as well as the application of painless and rapid rehabilitation concept, we believe that the criteria will be broadened as the number of clinical cases increases.

\section{List Of Abbreviations}

ASA: American Society of Anesthesiologists; IAAS: International Association for Ambulatory Surgery; CT: Computed Tomography; QOL: Quality Of Life; HIV: Human Immunodeficiency Virus; CM: centimeter; COVID-19: Corona Virus Disease 2019.

\section{Declarations}

\subsection{Ethics approval and consent to participate}

The principles of the Helsinki Declaration were followed in this study. The First Affiliated Hospital of Wenzhou Medical University and Taizhou Hospital of Zhejiang Province affiliated to Wenzhou Medical University ethics review committees approved this study. All of the patients have informed consent form before undergoing the operation.

\subsection{Consent for publication}

Not applicable.

\subsection{Availability of data and materials}

Data sharing is not applicable to this article as no datasets were generated or analyzed during the current study.

\subsection{Competing interests}

The authors declare that they have no competing interests.

\subsection{Funding}


The study was supported by the National Natural Science Foundation of China (No.81670695), Zhejiang Provincial medical and health technology program projects of China (Nos.2018PY031, 2018KY512, and 2019KY101), and Zhejiang Provincial Natural Science Foundation of China (Nos.LY14H180006 and WY2OH050001). These funds were used in the design of the study, data collection, and personnel support.

\subsection{Acknowledgements}

We are grateful to the medical staff helped this clinical study. This manuscript has been released as a pre-print at Research Square [21].

\subsection{Authors' contributions}

$\mathrm{H}-\mathrm{HJ}, \mathrm{X}-\mathrm{LZ}$ and S-JW had roles in the study conception and design, patient recruitment, data collection, data analysis, data interpretation, literature search, and writing of the manuscript. YB, W-LL, M-HJ, J-HL and X-LZ had roles in data collection, data analysis, and data interpretation. $\mathrm{H}-\mathrm{HJ}$ contributed to critical revision of the manuscript. All other authors contributed to data acquisition, data analysis, data interpretation, and reviewed and approved the final version.

\section{References}

1. Briggs C, Irving G, Mann C, Cresswell A, Englert L, Peterson M, Cameron I: Introduction of a Day-Case Laparoscopic Cholecystectomy Service in the UK: A Critical Analysis of Factors Influencing SameDay Discharge and Contact with Primary Care Providers. Annals of the Royal College of Surgeons of England 2009, 91:583-590.

2. Roberts L: Day surgery-National and international. Ambulatory Surgery 2006, 12:143-145.

3. Bas O, Nalbant I, Sener N, Firat H, Yeşil S, Zengin K, Yalcınkaya F, Imamoglu A: Management of Renal Cysts. JSLS : Journal of the Society of Laparoendoscopic Surgeons / Society of Laparoendoscopic Surgeons 2015, 19.

4. Eknoyan G: A Clinical View of Simple and Complex Renal Cysts. Journal of the American Society of Nephrology : JASN 2008, 20:1874-1876.

5. Agarwal M, Hemal A: Surgical Management of Renal Cystic Disease. Current urology reports 2011, 12:3-10.

6. Terada N, Ichioka K, Matsuta Y, Okubo K, Yoshimura K, Arai Y: The natural history of simple renal cysts. The Journal of urology 2002, 167:21-23.

7. Glassberg KI, Stephens FD, Lebowitz RL, Braren V, Duckett JW, Jacobs EC, King LR, Perlmutter AD: Renal dysgenesis and cystic disease of the kidney: a report of the Committee on Terminology, Nomenclature and Classification, Section on Urology, American Academy of Pediatrics. J Urol 1987, 138(4 Pt 2):1085-1092. 
8. Efesoy O, Tek M, Bozlu M, Doruk H: Comparison of single-session aspiration and ethanol sclerotherapy with laparoscopic de-roofing in the management of symptomatic simple renal cysts. Türk Üroloji Dergisi/Turkish Journal of Urology 2015, 41:14-19.

9. Yu W, Zhang D, He X, Zhang Y, Liao G, Deng G, Jin B: Flexible ureteroscopicmanagement of symptomatic renal cystic diseases. Journal of Surgical Research 2015, 196.

10. Mancini V, Cormio L, d'Altilia N, Benedetto G, Ferrarese P, Balzarro M, Defidio L, Carrieri G: Retrograde Intrarenal Surgery for Symptomatic Renal Sinus Cysts: Long-Term Results and Literature Review. Urologia Internationalis 2018, 101:1-6.

11. Busato W, Bettega L: Percutaneous Endocystolysis, a Safe and Minimally Invasive Treatment for Renal Cysts: A 13-Year Experience. Journal of endourology / Endourological Society 2010, 24:14051410.

12. Eissa A, Elsherbeny A, Martorana E, Pirola G, Puliatti S, Scialpi M, Micali S, Rocco B, Liatsikos E, Breda A et al: Non-conservative management of simple Renal cysts IN adults: A comprehensive review of literature. Minerva Urologica e Nefrologica 2018, 70:179-192.

13. Ryu D-S, Oh T-H: Laparoscopic Decortication of Large Renal Cysts: A Comparison Between the Transperitoneal and Retroperitoneal Approaches. Journal of laparoendoscopic \& advanced surgical techniques Part A 2009, 19:629-632.

14. Fabricant P, Seeley M, Rozell J, Fieldston E, Flynn J, Wells L, Ganley T: Cost Savings From Utilization of an Ambulatory Surgery Center for Orthopaedic Day Surgery. Journal of the American Academy of Orthopaedic Surgeons 2016, 24:1.

15. Rago R, Franceschini F, Tomassini C: Short Hospitalization System: A new way of interpreting day surgery care. Minerva anestesiologica 2014, 82.

16. Hemal A, Gupta N, Rajeev TP, Aron M, Bhowmik D, Jain R: Retroperitoneoscopic Managementof Infected Cysts in Adult Polycystic Kidney Disease. Urologia internationalis 1999, 62:40-43.

17. Gupta N, Goel R, Hemal A, Kumar R, Ansari M: Retroperitoneoscopic Decortication of Symptomatic Renal Cysts. Journal of endourology / Endourological Society 2005, 19:831-833.

18. Letica-Kriegel A, Salmasian H, Vawdrey D, Youngerman B, Green R, Furuya E, Calfee D, Perotte R: Identifying the risk factors for catheter-associated urinary tract infections: A large cross-sectional study of six hospitals. BMJ Open 2019, 9:e022137.

19. Boey W: Challenges in ambulatory surgery: discharge criteria. Annals of the Academy of Medicine, Singapore 1995, 24:906-909.

20. Tao R, Qu Z, Sun D, Deng Y, Mo Y, Chen J, Zhang Y, Xie X, Tang W, Liu W: Interpretation of clinical practice guideline for anorectal day surgery 2019 edition. Zhonghua wei chang wai ke za zhi = Chinese journal of gastrointestinal surgery 2019, 22:1118-1123.

21. Zhu X-L, Bano Y, Wu S-J, Jiang H-H: A Clinical Control Study on the Retroperitoneal Laparoscopy for Unilateral Simple Renal Cyst Decortication in the Day Ward; 2020.

\section{Tables}


Due to technical limitations, tables 1-4 are only available as a download in the Supplemental Files section.

\section{Figures}

\begin{tabular}{l} 
Exclusion \\
criteria(n=8) \\
(1) Age $>80$ years. \\
(n=3) \\
(2) A history of \\
previous \\
abdominal \\
surgery.( $\mathrm{n}=2$ ) \\
(3) Preoperative \\
American Society \\
of \\
Anesthesiologists \\
(ASA) grade was \\
III or IV, or \\
contraindications \\
to surgery or \\
anesthesia. (n=1) \\
(4) Imaging \\
suggested renal \\
cysts on both sides. \\
(n=1) \\
(5)The Bosniak \\
grade in imaging \\
was III or IV. \\
\hline
\end{tabular}

\begin{tabular}{|l|l|}
\hline \multicolumn{1}{|c|}{ Patients with surgical indications for renal cysts } \\
surgical indications(n=41)
\end{tabular}

\section{Figure 1}


Flow diagram of study design and participants.

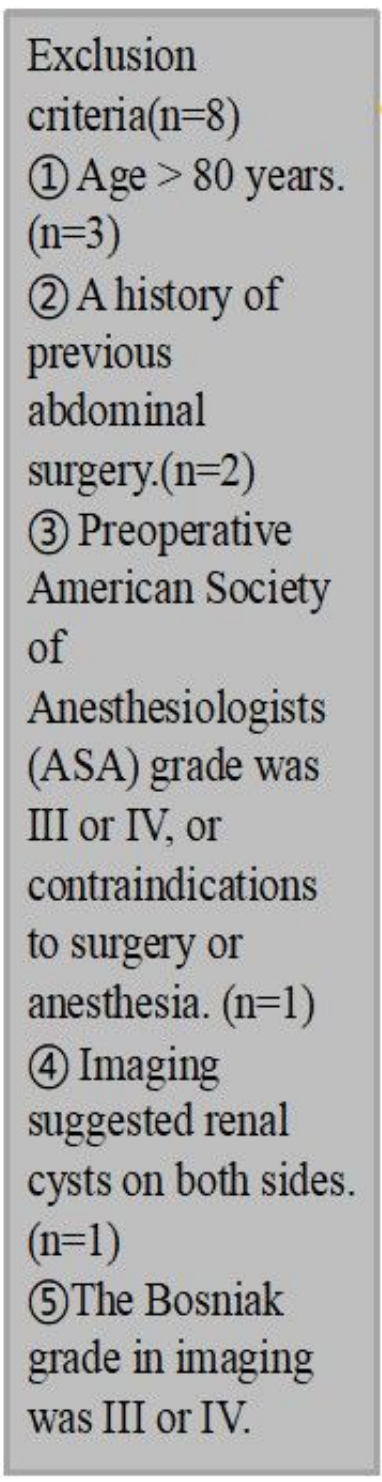

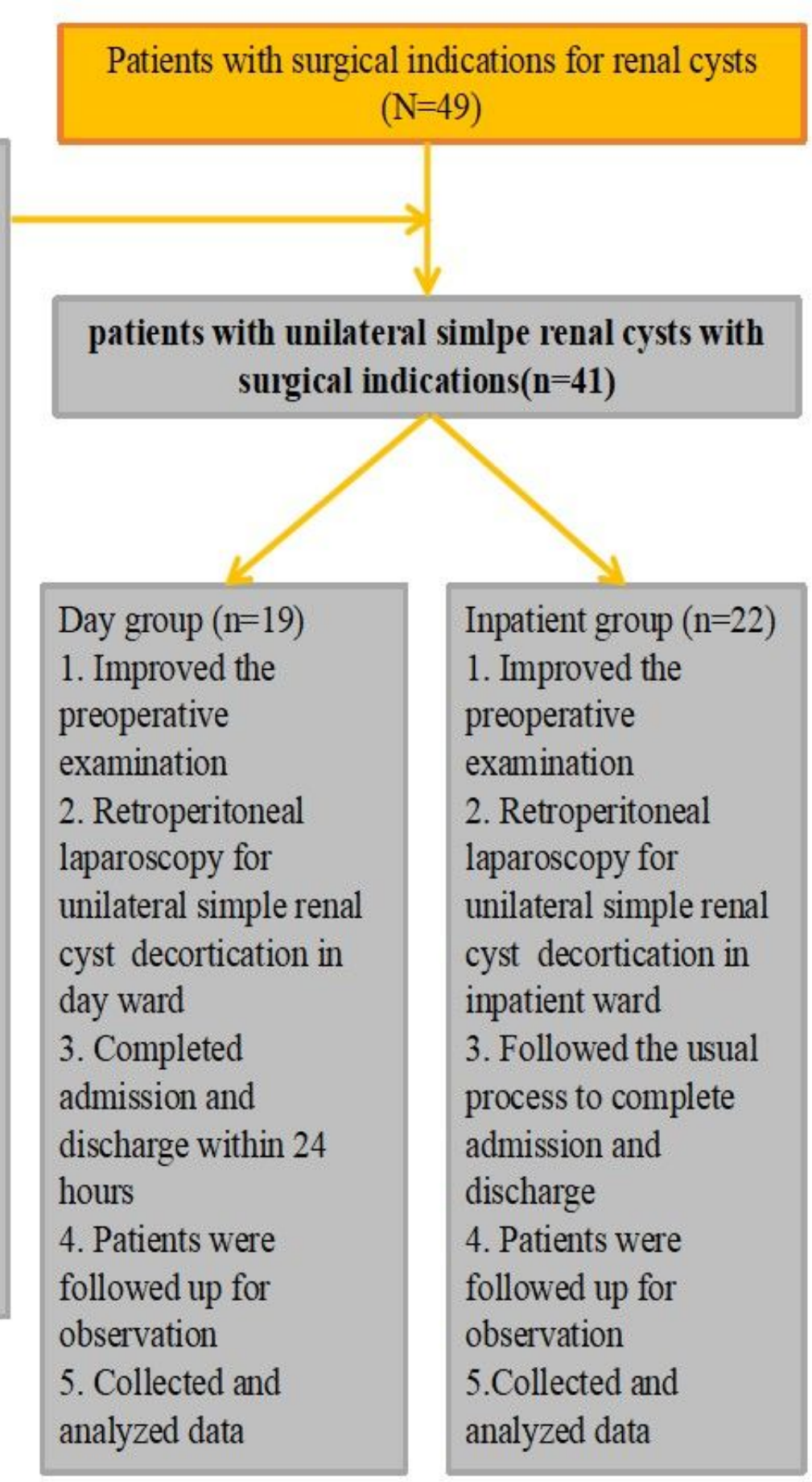

\section{Figure 1}

Flow diagram of study design and participants.

\section{Supplementary Files}


This is a list of supplementary files associated with this preprint. Click to download.

- Table1.xls

- Table1.xls

- Table2.xls

- Table2.xls

- Table3.xls

- Table3.xls

- Table4.xIs

- Table4.xls 